\title{
Actitudes del gremio médico hacia las prácticas profesionales de las trabajadoras y los trabajadores sociales sanitarios
}

\author{
Pedro Daniel Martínez Sierra ${ }^{1}$
}

Recibido: 02/12/2019 / Revisado: 12/12/2019/Aceptado: 03/03/2020

Resumen. El presente artículo tiene como objetivo: analizar las actitudes del gremio médico respecto a las prácticas profesionales de las/los trabajadores sociales sanitarios. Para su desarrollo se ha utilizado la teoría de las representaciones sociales de Serge Moscovici, por ser una opción con herramientas conceptuales y metodológicas que permiten una aproximación a las actitudes de las/los sujetos. Es un estudio cualitativo, donde las técnicas e instrumentos empleados para la recuperación de los datos empíricos han sido entrevista y cuestionario, dirigidos a médicos/as de base y residentes de las siguientes especialidades: medicina interna, pediatría, ginecología, oncología, terapia intensiva y patología del Hospital General de México, institución pública de segundo nivel de atención en salud. Los hallazgos del estudio dieron cuenta, de que, para los médicos, la práctica de las/los trabajadores sociales se reduce al tareas de carácter administrativo, lo que no implica necesariamente una actitud negativa hacia este ejercicio profesional. Consideran a los trabajadores sociales un grupo importante en el movimiento hospitalario, con una alta carga de trabajo que resuelve diversos procesos burocráticos. Sin embargo, no los reconocen como grupo de profesionales que puede aportar un conocimiento científico para mejorar la salud de las/los pacientes.

Palabras claves: actitudes, representaciones sociales, médicos, trabajadores sociales, práctica profesional sanitaria

Sumario: Introducción. 1. Marco teórico. 2. Metodología. 2.1 Diseño y contexto. 2.2 Participantes. 2.3 Procedimientos y materiales. 2.4 Criterios éticos del estudio. 2.5 Análisis de datos. 3. Resultados y discusión. 4. Conclusiones. 5. Referencias bibliográficas.

Cómo citar: Martínez Sierra, P. D. (2020) Actitudes del gremio médico hacia las prácticas profesionales de las trabajadoras y los trabajadores sociales sanitarios. Cuadernos de Trabajo Social, 33(1), 313-325.

\section{Introducción}

El presente estudio forma parte de la tesis de doctorado generada en el Programa de Posgrado en Pedagogía de la Universidad Nacional Autónoma de México (UNAM), que llevo por título Los médicos y sus representaciones sociales sobre la práctica profesional de los trabajadores sociales, cuyo objetivo consistió en analizar las representaciones sociales de estos profesionales sanitarios sobre el objeto delimitado. Para su presentación como artículo científico se retoma una de las dimensiones de esta teoría: actitud, con el propósito de presentar un análisis profundo de este objeto desde el corpus teórico de esta perspectiva.

Como parte de la fundamentación, se localizó un número limitado de estudios que recuperan la mirada de otro grupo profesional sobre la práctica de las/los trabajadores sociales, destacando los trabajos de: Muriel et al., (2018), Minetto y Voragini, (2017), Rosacher (2015) y Pérez (2013), desarrolladas en distintos campos de actuación y que muestran como médicos, psicólogos y profesores asocian las prácticas de las/los trabajadores sociales con acciones filantrópicas o de caridad que dieron origen a esta carrera.

\footnotetext{
Escuela Nacional de Trabajo Social, Universidad Nacional Autónoma de México, México.

ms.pedrodaniel@comunidad.unam.mx
} 
Por su parte, Pérez (2005), Barrantes, (2003) y otros estudios mostraron una serie de críticas y descalificaciones relacionadas con la falta de reconocimiento, inmediatez y carencia de reflexividad en los servicios proporcionados por las/los trabajadores sociales; asimismo, plantearon desafíos que deben enfrentarse en materia de formación académica, identidad colectiva y proyección, reflejándose una búsqueda insistente por la identidad o la resignificación de la actividad profesional, siendo necesario no solo analizar su reflexión respecto a su práctica, sino recuperar la mirada de otros grupos con los que se ha vinculado a lo largo de su historia. En las investigaciones de Martínez (2014), Pérez (2005) y Barrantes (2003) rescatan no solo lo que piensan las/los trabajadores sociales de sí mismos, sino también lo que otros sujetos creen de ellos, con el fin de reflexionar sobre su identidad y evidenciar características que los diferencian de otras carreras.

Para contribuir con esta línea de estudio, se presenta un artículo que busca develar los pensamientos de sentido común de otros grupos profesionales respecto a la práctica profesional de las/los trabajadores sociales, para ello, fue seleccionado el ámbito sanitario, referido como el campo de mayor implantación e historia, en donde principalmente licenciados, técnicos y estudiantes de esta carrera por más de cuarenta años han desarrollado su ejercicio y prácticas escolares en vinculación con otros grupos sanitarios.

En el caso de México, este ámbito se rige bajo la norma de la Ley General de Salud. El servicio público lo integran el Instituto Mexicano del Seguro Social (IMSS), Instituto de Seguridad y Servicios Sociales de los Trabajadores del Estado (ISSSTE), Petróleos Mexicanos (PEMEX), Secretaría de la Defensa (SEDENA) y Secretaría de Marina (SEMAR) y otros. Así mismo, existen otras instituciones o programas que atienden a la población carente de seguro social como los hospitales que ofrecen los servicios de la Secretaría de Salud (SSA), el Seguro Popular de Salud (SPS) y otros.

El área de la salud se fracciona en tres niveles de atención: el primero está constituido por Unidades de Medicina Familiar, Centros de Salud y Clínicas Familiares, donde se proporcionan servicios básicos y acciones de contacto inicial o preventivas. El segundo lo integran hospitales federales, generales, regionales, integrales y comunitarios, donde se atienden a pacientes remitidos del primer nivel que requieren de procedimientos diagnósticos, terapéuticos y de rehabilitación. El tercero lo conforma una red de hospitales de alta especialidad que brindan atención a enfermedades complejas y de alto riesgo que demandan de una vigilancia más específica por el deterioro que provocan en la salud de las/los pacientes.

Las problemáticas que presentan los usuarios requieren diversos profesionistas con un alto nivel de formación, experiencias, conocimientos y campos de dominio. La Organización Mundial de la Salud (OMS, 2017) refiere que el personal sanitario está integrado por todas aquellas personas que desarrollan tareas cuya principal objetivo es promover la salud. El equipo está conformado por: médico cirujano, médico de distintas especialidades, médico residente, médico pasante, médico interno, enfermeras, odontólogos, nutricionistas, terapeutas, psicólogos, trabajadores sociales y camilleros. El equipo administrativo lo integran: asistentes médicos, recepcionistas, secretarías, archivistas, personal de farmacia, vigilancia, limpieza y otros.

El ámbito sanitario es reconocido como la principal fuente de empleo de las/los trabajadores sociales mexicanos al localizarse su mayor cantidad de plazas validadas por el Estado. De acuerdo con Vázquez y Cid de León (2015), del 80\% de estos profesionistas que trabajan en dicho sector, el $56 \%$ tiene una plaza del Estado, y el resto de tipo federal. El personal señalado se encuentra ubicado esencialmente en hospitales de segundo nivel de atención con el $49.2 \%$, seguido de institutos de alta especialidad de tercer nivel con el $32.2 \%$, y finalmente clínicas de medicina familiar con el $16.9 \%$. Su perfil en el ámbito sanitario se ha vinculado primordialmente con actividades de educación social relacionadas con la ejecución de modelos preventivos, acciones de capacitación dirigidas a pacientes, familia y comunidad para el restablecimiento de la salud.

Ahora bien, las instituciones donde médicos/as y trabajadores/as sociales han ejercido su práctica poseen una estructura jerárquica, que establece relaciones de coordinación y subordinación correspondiente a la organización general de los servicios de salud. Por lo que no resulta extraña la diferenciación salarial del médico/a con otras profesiones debi- 
do al aumento de especialidades, la incorporación de equipos sofisticados y la complejidad de las enfermedades. Con base en ello, la Secretaria de Salud (2017), fracciona al personal de la siguiente forma: la adscripción médica se divide en dos, la primera corresponde a médico especialista y médico general en área normativa "A", "B", "C" y "D", categorías asignadas a trabajadores de confianza con puestos de dirección, jefatura o coordinación. La segunda está dirigida a quienes se encuentran en ámbitos operativos. En el caso de las/los trabajadores sociales desempeñan cargos de: Supervisora de trabajo social en área médica y técnica en trabajo social, clasificados en "A", "B" y "C", claves dirigidas a personal con estudios de licenciatura y técnico, sin considerarse en una denominación específica a quienes cuentan con estudios de posgrado.

\section{Marco teórico}

Para el desarrollo de este estudio se utilizó la teoría de las representaciones sociales perspectiva que en el campo de la investigación social ha sido empleada como una de las opciones teóricas y metodológicas de actualidad que explican cómo los sujetos ven y dan sentido a su realidad, según su propio sistema de referencias. Su uso permite comprender cómo se constituye y organiza el conocimiento de sentido común que autoriza a los individuos tomar decisiones o definir sus comportamientos en la vida cotidiana.

La teoría de las representaciones sociales fue acuñada por el filósofo de la ciencia, de origen rumano, Serge Moscovici, a través de su tesis doctoral La psychanalyse, son image, son public en el capítulo I de su libro La representación social: un concepto perdido, advertía sobre el problema de definir esta noción, al señalar que, si bien resulta fácil captarlas por ser entidades casi tangibles que "circulan, se cruzan y se cristalizan sin cesar en nuestro universo cotidiano a través de una palabra, un gesto, un encuentro el concepto no lo es" (Moscovici, 1979, p. 27). Una de las primeras definiciones que ofrece sobre representaciones sociales:

Es un corpus organizado de conocimientos y una de las actividades psíquicas gracias a las cuales los hombres hacen inteligible la realidad física y social, se integran en un grupo o en una relación cotidiana de intercambios, liberan los poderes de su imaginación ( p.18).

Las representaciones sociales designan, a la vez. un proceso y un contenido integrado por opiniones, conocimientos, creencias propias de una cultura en relación a objetos del medio social. Una vía para tener clara esta distinción son las dimensiones de las representaciones sociales, según Moscovici: se organizan de maneras diversas según las clases, las culturas o los grupos y constituyen tantos universos como clases, culturas o grupos existentes. Cada universo, según nuestra hipótesis tiene tres dimensiones, la actitud, la información y el campo de representación o la imagen ( $\mathrm{p}$. 45).

- La información es definida como una "-dimensión o concepto-que se relaciona con la organización de los conocimientos que posee un grupo con respecto a un (objeto social)" (p. 45).

- $\quad$ El campo de representación es la noción más compleja, expresa la idea de la organización del contenido: hay campo de representación allí donde hay una unidad jerarquizada de los elementos, pero también el carácter más o menos rico de este contenido, las propiedades propiamente cualitativas, imagina- tivas de la representación" (Herzlich, 1975, p. 400).

- En el caso de la actitud que interesa analizar en este estudio, es considerada como las más frecuente de las anteriores y, quizás la primera desde el punto de vista genético, así la información, sea reducida o exista un campo de representación poco organizado, se debe a que hay información previa y representamos una cosa después de haber tomado una posición que expresa una orientación general, positiva o negativa, frente al objeto de representación (Herzlich, 1975; Moscovici, 1979).

Dicha teoría proporcionó directrices para el análisis de las actitudes de las/los médicos y la red de significaciones que han edificado sobre el objeto de representación, al facilitar un acercamiento al entorno social, conducta, interacciones y prácticas. 


\section{Metodología}

\subsection{Diseño y contexto}

Para el desarrollo de este estudio fue indispensable inscribir el objeto en una perspectiva epistemológica para delinear una ruta de indagación e interpretación. En ese sentido, se eligió el constructivismo que reclama un abordaje desde el pensamiento del sujeto, lo que dirigió la mirada hacia una de las escuelas de la teoría de las representaciones sociales, la sociogenética o procesualista que, según Banchs (2000) presenta una orientación cualitativa al interesarse por la compresión del sentido, producciones simbólicas e interacciones.

La posición epistemológica y metodológica fueron elementos articuladores que orientaron las aproximaciones para analizar la realidad de interés, lo que llevo a la definición del sujeto, objeto y escenario, así como a las estrategias y herramientas para obtener información de relevancia que coadyuvará al desarrollo del estudio.

En ese sentido, los criterios para definir el escenario fueron: presencia de médicos/as y trabajadoras/es sociales, posibilidades de ingreso, intereses del investigador y redes de apoyo para el acceso a la institución; siendo seleccionado el Hospital General de México "Dr. Eduardo Liceaga" de la Ciudad de México (CDMX), pertenece a la Secretaría de Salud (SSA). Es una institución pública, descentralizada del Gobierno Federal, cuenta con una de las plantillas más numerosas de trabajadores/ as sociales y médicos/as (1,222 médicos/as y 116 trabajadores/as sociales), ya que atiende a más de 70 mil pacientes al año. La relación entre estos profesionistas se da en las más de 44 unidades del hospital, que son servicios médicos de alta especialidad.

Lograr el ingreso a esta institución tan trascendente en la historia de la medicina mexicana, no fue una tarea sencilla, incluso fue considerado como uno de los grandes retos del estudio. Para superarlo fue necesario inscribir este estudio como protocolo para dictaminación ante los "Comités de Ética e Investigación", integrados por médicos/as que trabajan principalmente líneas de investigación biomédicas, epidemiológicas, clínicas y otras. El proyecto se registró como estudio sociomédico, categoría donde ingresan principalmente investigaciones provenientes de ciencias sociales y humanidades.

\subsection{Participantes}

\subsubsection{Tipo de muestreo}

La recogida de la información se realizó a partir de un muestreo intencional o de conveniencia donde la selección de las/los participantes se hizo de acuerdo con los objetivos de la investigación; se eligieron médicos/as del Hospital General de México por el papel de autoridad, fuente de poder y conocimiento, desempeñados en el ámbito hospitalario.

\subsubsection{Criterios de inclusión y exclusión}

Se construyeron con base en las posibilidades de ingreso al campo, de modo que el proceso estuvo definido en gran medida por las decisiones de las autoridades (se les aplicó el cuestionario a 132 médicos/as, y de este universo se desarrollaron entrevistas con 8). Sin embargo, hubo algunas posibilidades que permitieron delinear las características de la población. Por lo tanto, se definieron los siguientes criterios para la selección de los participantes:

- Médicos/as con especialidades vinculadas a la práctica profesional del trabajador social.

- Médicos/as residentes o de base del Hospital General de México.

- Médicos/as con mínimo un año de antigüedad en el hospital.

- Médicos/as interesados en participar en el estudio.

\subsection{Procedimiento y materiales}

En un primer momento se utilizó un cuestionario semiestructurado como una vía de entrada con grupos grandes como las/los médicos del Hospital General de México, su diseño ofreció la posibilidad de obtener información sistemática y ordenada que pudo ser profundizada a través de la entrevista. Para su elaboración se consideraron las características de la población hacia la cual fue dirigida: un profesionista altamente demandado, con limitaciones de tiempo y extenuantes jornadas de trabajo; lo que llevó a estructurarlo en dos cuartillas y con preguntas principalmente cerradas que pudieran responderse en máximo 10 minutos. El instrumento estuvo conformado por dos secciones: datos generales y cuatro preguntas de opción 
múltiple trabajadas desde la dimensión de actitud como se muestra en el cuadro 1.

Cuadro 1. Diseño de preguntas del cuestionario

\begin{tabular}{|l|c|l|}
\hline \multicolumn{1}{|c|}{ Preguntas } & $\begin{array}{c}\text { Categorías de } \\
\text { análisis }\end{array}$ & $\begin{array}{l}\text { Fuentes de información para las } \\
\text { opciones de respuesta }\end{array}$ \\
\hline $\begin{array}{l}\text { Seleccione el profesionista que considere po- } \\
\text { dría ser prescindible en el hospital. }\end{array}$ & Actitudes & $\begin{array}{l}\text { Hospital General de México } \\
\text { (HGM, 2016). Cuaderno Estadísti- } \\
\text { co de enero a septiembre del HGM. }\end{array}$ \\
\hline $\begin{array}{l}\text { ¿Cuál cree que es el nivel de estudios de la } \\
\text { mayoría de las/los trabajadores sociales en } \\
\text { este hospital? }\end{array}$ & Actitudes & $\begin{array}{l}\text { López et al., (2007). El Trabajo So- } \\
\text { cial en México. }\end{array}$ \\
\hline $\begin{array}{l}\text { ¿Cuál es la importancia que considera tiene la } \\
\text { práctica profesional de las/los trabajadores so- } \\
\text { ciales en el hospital? }\end{array}$ & Actitudes & $\begin{array}{l}\text { López et al., (2007). El Trabajo So- } \\
\text { cial en México. }\end{array}$ \\
\hline $\begin{array}{l}\text { ¿ué tan preparados considera que están } \\
\text { las/los trabajadores sociales de este hospital } \\
\text { para enfrentar la problemática social de los } \\
\text { pacientes? }\end{array}$ & Actitudes & $\begin{array}{l}\text { López et al., (2007). El Trabajo So- } \\
\text { cial en México. }\end{array}$ \\
\hline
\end{tabular}

Fuente: Elaboración propia con base en las fuentes de información descritas en la columna tres.

En total se distribuyeron 132 cuestionarios, 105 con residentes y 27 con personal de base que fueron aplicados de la siguiente forma:

- En la primera fase del trabajo de campo, las autoridades del hospital asignaron grupos de médicos/as residentes de las especialidades en: medicina interna, oncología, pediatría, ginecología y radiología. La aplicación del cuestionario se realizó durante cinco días no consecutivos, en los horarios de clase establecidos por la institución.

- Con la población de médicos/as de base, la aplicación se llevó a cabo en tres días no continuos, con una permanencia de cuatro horas por cada uno. Para realizar esta actividad fueron identificados lugares estratégicos que concentraran a este personal como: pabellones, cafeterías, pasillos, auditorios e incluso estacionamientos.

La segunda fase del trabajo de campo consistió en el desarrollo de entrevistas como una vía de indagación en aspectos que el cuestionario no integra; fue de tipo enfocada pero no a profundidad, debido a las limitaciones de tiempo que presenta la población. Debe señalarse que 22 médicos/as aceptaron a través del cuestionario que se les realizará una entrevista; no obstante, solo se concretaron ocho: cinco con residentes y tres con personal base de las especialidades de medicina interna, pediatría, ginecología, oncología, terapia intensiva y patología. Esta técnica de investigación estuvo acompañada de una guía de entrevista semiestructurada integrada por cinco preguntas que se muestran en el cuadro 2. 
Cuadro 2. Diseño de preguntas de la entrevista

\begin{tabular}{|c|c|c|}
\hline $\begin{array}{l}\text { Categoría de } \\
\text { análisis }\end{array}$ & Preguntas & $\begin{array}{l}\text { ¿Qué se pretende } \\
\text { encontrar? }\end{array}$ \\
\hline $\begin{array}{l}\text { Actitud sobre } \\
\text { la práctica pro- } \\
\text { fesional de las/ } \\
\text { los trabajadores } \\
\text { sociales. }\end{array}$ & $\begin{array}{l}\text { - ¿Considera importante que haya profesionistas como las/los } \\
\text { trabajadores sociales en este hospital y por qué? } \\
\text { ¿Cree que la actividad profesional que desarrollan las/los tra- } \\
\text { bajadores sociales debería ir más allá de lo que actualmente } \\
\text { realizan? } \\
\text { - ¿Qué tan preparados considera que están las/los trabajadores } \\
\text { sociales para desarrollar las funciones que se les ha asignado } \\
\text { en este hospital y por qué? } \\
\text { ¿Cree que se les brinda la importancia suficiente a las acti- } \\
\text { vidades profesionales desarrolladas por las/los trabajadores } \\
\text { sociales en este hospital? } \\
\text { ¿Cómo describiría la práctica profesional de las/los trabaja- } \\
\text { dores sociales de este hospital? }\end{array}$ & $\begin{array}{l}\text { Valoraciones po- } \\
\text { sitivas o negativas } \\
\text { sobre la práctica } \\
\text { profesional que } \\
\text { realizan los traba- } \\
\text { jadores sociales }\end{array}$ \\
\hline
\end{tabular}

Fuente: Elaboración propia con base en las dimensiones de actitud e información de las representaciones sociales de Moscovici (1971).

Las entrevistas se efectuaron en el día, horario, margen de tiempos señalados por las/los médicos al hacerles la invitación. Éstas fueron grabadas en audio con su previa autorización, se desarrollaron en las instalaciones del hospital (oficinas y áreas comunes), las cuales tuvieron un papel evocativo para la expresión de sus ideas, informaciones, opiniones, conocimientos y experiencias sobre la práctica profesional de las/los trabajadores sociales. Debe señalarse que únicamente se logró un encuentro con las/los médicos de 8 a 25 minutos, debido a las limitaciones de su tiempo.

\subsection{Criterios éticos del estudio}

Previo a la aplicación del cuestionario y desarrollo de las entrevistas, se establecieron algunas consideraciones éticas, con el propósito de generar un ambiente de confianza en la búsqueda del conocimiento, así como un respeto por la integridad de los médicos/as (Kavle, 2011). Por ello, se les informó lo siguiente: el objetivo de la entrevista y cuestionario; la utilización de códigos para resguardar la confidencialidad; el uso de sus datos personales y respuestas se usaran para fines del estudio; la transcripción de su entrevista a quienes lo soliciten les llegaría vía correo electrónico para que autoricen su uso y validen el contenido; además se contempla realizar publicaciones sobre este tema garantizándoles el anonimato.

\subsection{Análisis de datos}

Para la recogida de los datos se utilizó el método inductivo dado que la investigación no buscó examinar los resultados con fines estadísticos y probabilísticos, ni generalizar los hallazgos en el grueso de la población, sino desarrollar un análisis profundo de las expresiones, lo que no excluye el usos de instrumentos como un cuestionario semiestructurado, que no forman parte intrínseca de alguna epistemología. Ya lo refiere Ducoing (2016), los positivistas no son propietarios de los números, ni los interpretativistas de las cualidades, por lo tanto, se realizó una selección basada en el planteamiento del problema, el referente metodológico y las características de la población.

En ese sentido, los resultados del cuestionario fueron procesados en una base de datos diseñada en el programa Statistical Package for the Social Science (SPSS) donde se asignaron códigos aleatorios, del 1 al 8, en función a las opciones de respuesta, con la alternativa 'omitió' para quienes seleccionaron más de una o no contestaron.

En el caso de las entrevistas su tratamiento fue el siguiente: Se transcribieron en un procesador de textos de Word, los testimonios seleccionados fueron presentados con fines de confidencialidad y organización de la información utilizando los siguientes códigos: (E) entrevista y (\#) número de folio. Con el propósito 
de identificar el tipo de médico se utilizó (MB) para médico base; (MR) en el caso del residente seguido del año de avance en la especialidad (\#). Ejemplos: E5-MB y E3-MR3.

Una vez transcrita, la información fue sistematizada en el programa de análisis cualitativo Atlas ti donde se abrió una unidad hermenéutica que permitió utilizar algunas de sus herramientas como: el administrador de documentos primarios; administrador de familias y administrador de códigos, finalmente con base en la orientación epistemológica y metodológica del estudio, se procedió a la interpretación siendo seleccionado como modo de análisis la condensación del significado que integró tres momentos:

\subsubsection{Unidades de sentido}

Se determinaron las unidades de significado a partir de las palabras o frases repetidas con insistencia por los médicos, las cuales guardan un vínculo con la dimensión de actitud.

\subsubsection{Formulación de una manera directa}

Se reelaboró en temas centrales lo que fue denominado como 'una unidad de significado natural', misma que fue organizada temáticamente a partir de la comprensión de las declaraciones las/los sujetos, lo que implicó el registro de las primeras interpretaciones.

\subsubsection{Interrogación de unidades de significado}

Con base en el propósito del estudio fueron interrogadas las unidades de significado desde el marco de referencia.

\section{Resultados y discusión}

La pregunta detonadora que permitió recuperar la importancia, nimiedad o indiferencia que las/los médicos conceden a la práctica fue: ¿cuál es la importancia que tiene la práctica profesional de las/los trabajadores sociales en el hospital? A partir de ella surgieron otros cuestionamientos como: ¿qué tan preparados considera que están las/los trabajadores sociales de este hospital para enfrentar la problemática social de los pacientes?, ¿considera importante que haya profesionistas como las/ los trabajadores sociales en este hospital y por qué?, ¿cree que se les brinda la importancia suficiente a las actividades profesionales desarrolladas por las/los trabajadores sociales en este hospital?

El componente actitudinal en los discursos médicos/as estaba vinculado con valores y normas con los cuales tradicionalmente han sido formados: alto sentido de competencia, responsabilidad, eficiencia, confidencialidad y otros. Sus posturas respecto al objeto se manifestaron desde su lugar jerárquico en el Hospital General de México, correspondiente al de un gremio de élite con arraigo en la tradición de su ejercicio, que utiliza criterios de distinción frente a otras profesiones de la salud.

En la opinión de médicos/as, el gremio de Trabajo Social está encasillados en una posición subalterna, al considerarlos parte del personal administrativo que proporciona servicios sociales, y no como profesionistas del área de la salud con autoridad o saberes específicos que contribuyen en la atención de aspectos psicosociales del paciente.

Al Trabajo Social se le relaciona con otras ciencias, como medicina y, sin embargo, se ha preocupado poco por la construcción de sus propios referentes científicos. Basta con revisar sus planes de estudio en Latinoamérica, integrados por una importante cantidad de asignaturas provenientes de otros campos disciplinares. La posición de consumidora de saberes y la falta de especialización en el campo sanitario mexicano son elementos que condicionan su posición en la institución. $\mathrm{Su}$ participación en algunos servicios se ha reducido solo al llenado de instrumentos, brindándole a su práctica una categoría administrativa.

Los atributos incorporados culturalmente a la investidura médica les han otorgado una visión particular que tiende a valorar, diagnosticar y proponer alternativas sobre diversas situaciones. De ahí que en los discursos de médicos/as de base con cargos de coordinadores, sobresalieran "evaluaciones" que, aunque de orden primario, señalaron la forma en cómo debería ser la práctica profesional de las/los trabajadores sociales. He aquí un ejemplo:

Debería haber especialidades como en la enfermería que hay residencias o especialidades, así debería suceder en trabajo social, para terapia intensiva, para urgencias, para voluntariado, porque son cosas totalmente distintas. (E2-MB). 
Ante la falta de reconocimiento de un conocimiento especializado en las/los trabajadores sociales, el gremio médico utilizó expresiones con una connotación ordenativa como: "debería de...", , "requiere...,", "le falta...", "no conoce...","solo sirven..." "y su principal función es...", las cuales evidenciaron una construcción discursiva desde una posición autoritaria, con rango superior al de este profesionista.
Cuando se pregunto a los médicas/os cuál de los siguientes profesionistas: psicólogos, trabajadores sociales, enfermeras, nutriólogos, odontólogos, médicos u otros podrían ser prescindibles se obtuvo que el $69 \%$ de las/los médicos refirió que ninguno (véase Gráfico 1), pues en las últimas décadas se ha pasado de una centralización de la asistencia médica a una socio-sanitaria, es decir, otros grupos profesionales se han integrado.

Gráfico 1. Seleccione el profesionista que considere prescindible en el hospital

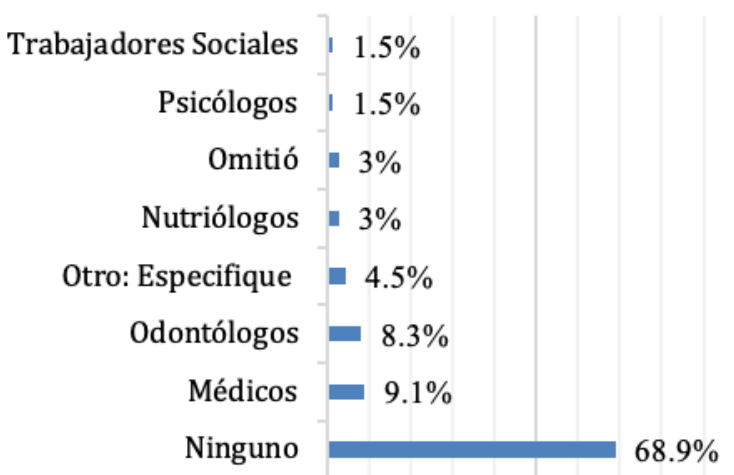

Fuente: Elaboración propia con base en los resultados del cuestionario.

Al no mencionar a las/los trabajadores sociales como un grupo prescindible, se anticipó una actitud positiva y aprobatoria. Son considerados piezas clave del movimiento hospitalario al ser el primer contacto de atención sanitaria, principalmente con grupos de bajas posibilidades económicas de pago.

Para médicas/os, las acciones de asistencia y ayuda al necesitado son la razón de ser de la práctica de trabajadores/as sociales en el hospital. Algunas/os por su formación biopsicosocial están más sensibilizados con las problemáticas sociales de las/los pacientes, por ello valoran la importancia de las/los trabajadores sociales al intégralos en discusiones sanitarias.

Las/los médicos manifestaron que la plantilla de trabajadores sociales era limitada, debido a las necesidades sociales de las personas, mas no ven como prioritario su aumento. Pese al incremento de las demandas sociales, el crecimiento de la plantilla de trabajadores sociales ha sido escaso; de 2016 a 2017 en el Hospital General de México solo se generaron 4 nuevas plazas aún con la excesiva carga de trabajo y saturación que tienen durante la realización de sus labores cotidianas.

Debido a la demanda de servicios sanitarios, la institución ha destinado oficinas específicas para el desarrollo de la práctica de las/los trabajadores sociales, por ser el primer contacto con la población para evaluar su capacidad de pago. Esta área se encarga de concentrar, registrar y cuantificar mensualmente, el total de clasificaciones socioeconómicas. Por ello, dentro de las instalaciones del Hospital General de México, se cuenta con un pabellón exclusivo de Trabajo Social en el área de consulta externa, integrado por más de 10 oficinas, donde se aprecian filas interminables de pacientes y familiares durante todo el día.

Asimismo, las/los médicos identificaron que las/los trabajadores sociales tienen una carga excesiva de trámites administrativos que limita su participación en el trabajo interdisciplinario, pero estas actividades resultan indispensables en instituciones de salud pública. Para ellos, es importante que exista personal con este perfil en la institución, porque son resolutivos. Cuando se les preguntó "si no hu- 
biera trabajadoras/es sociales en el hospital, ¿quiénes podrían desempeñar esta función?” Algunos refirieron que podrían ser secretarias u otro trabajador capacitado en el área social, otorgándole solo un valor administrativo a su práctica.

Aunque el 54\% de las/los médicos reconocen que la práctica de las/los trabajadores sociales tiene mucha importancia, y otro 38\% la considere moderada, no se considera como una práctica con algún tipo de centralidad, como se muestra en el Gráfico 2. Aunque reconocen que las condiciones sociales influyen en el tratamiento de las/los pacientes, no tienen un peso prioritario en sus diagnósticos médicos.

Gráfico 2. Importancia que considera tiene la práctica profesional de las/los trabajadores sociales en el hospital

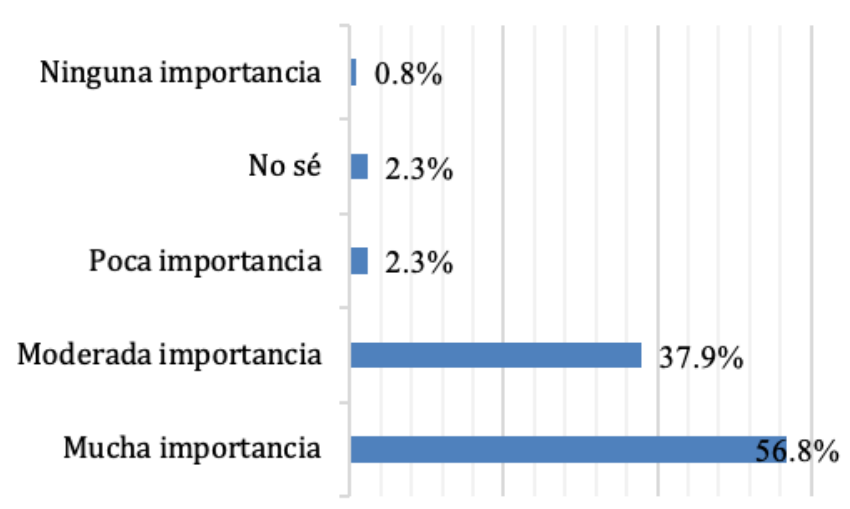

Fuente: Elaboración propia con base en los resultados del cuestionario.

Por otro lado, debe señalarse que de los integrantes del equipo sanitario, las/los trabajadores sociales son quienes cuentan con una de las más bajas retribuciones económicas, que los coloca en una clara desventaja profesional frente a otros grupos. Sus percepciones corresponden a una formación técnica y de licenciatura, aunque algunos cuentan con estudios de posgrado, al respecto un médico de base de 22 años de experiencia en esta institución:

A un trabajador social le pagan prácticamente como un obrero, o sea como un trabajador asalariado, que bueno, todos somos asalariados, pero no se le da el valor que en realidad tiene el trabajador social, cuando digamos que a los médicos o los químicos o las enfermeras tienen, supongamos, un sueldo de diez mil pesos mensuales, al trabajador social le pagan seis mil o cinco mil, o sea no ganan a la par de otras profesiones, y no nada más es en las instituciones públicas también en las privadas (E7-MB).

El nivel jerárquico de las profesiones es un rasgo relevante que delimita una forma de relación en el interior de las instituciones. Lo señalado corresponde a que médicos/as poseen los niveles educativos más altos del personal sanitario, la mayoría cuenta con estudios de posgrado (especialistas o subespecialistas). Las disimilitudes entre los grados académicos y categorías salariales entre los profesionistas en cuestión, son factores que influyen en su actitud y trato, además en la consideración de sus opiniones en temas de su competencia, aquí se muestra en este testimonio de un médico de base:

El trabajador social puede decir: "oigan, fíjense que yo estado viendo que en urgencias los pacientes tienen una estancia de espera de dos días, y no hay condiciones óptimas para su espera". Puede incluso tener las evidencias de que el paciente está ahí tirado, con una cobija, y sin sillas, pero no hay una injerencia que pueda decir: "yo trabajador social vi esto", y que pese su opinión para modificar esas condiciones (E2-MB).

La posición jerárquica de los/as médicos/ as es símbolo de mando, responsabilidad y autoridad; pese a que han cedido algunos es- 
pacios a otros grupos, aún concentran la cúpula de poder en los hospitales. Su liderazgo les posibilita la toma de decisión sobre temas de atención prioritaria. Estos profesionistas se han suscrito con facilidad en los puestos de mayor rango en el hospital: direcciones, subdirecciones, coordinaciones, jefaturas, áreas de investigación, cargos operativos y de administración de los servicios de salud; pero grupos como las/los trabajadores sociales han tenido accesos restringidos a dichos espacios decisivos, al no proyectar un ejercicio de autoridad profesional. Por consiguiente, la dinámica organizacional es uno de los condicionamientos que limita la posibilidad de que las/los trabajadores sociales aspiren a nombramientos distintos a los de su categoría y que ello tenga efecto en su reconocimiento profesional.

Otro de los factores que impacta en esta falta de reconocimiento es que la plantilla del personal de trabajadoras/es sociales está integrada principalmente por mujeres. De acuerdo con Lorente (2018), la condición de género en el ejercicio laboral ha limitado su acceso a cargos de alta responsabilidad dentro de las instituciones; sus funciones, al estar asociadas con acciones de ayuda y cuidado hacia los demás, están fuera de lo que se considera como "tareas de prestigio" para prosperar laboralmente.

Esta posición de subalternidad condiciona la visibilización de otras habilidades y dificulta su reconocimiento; al no tener presencia en plazas de mayor jerarquía, genera un encasillamiento que alimenta prejuicios y estereotipos sobre su rol, así se señala en esta declaración de un médico de base:

Jerárquicamente lo que te dicta como médico que alguien es superior a ti, es por nombramiento o académicamente, $\mathrm{y}$ es lo que trasciende y lo que importa, y es a lo que se le da peso, a menos que falte dinero o material, volteamos a ver al que paga o al que compra los insumos, pocas veces nos plantean que el trabajador social puede hacer mucha injerencia, tanto con el paciente como con todas las áreas de poder del médico, lo encasillan como paciente problemático que se vaya a trabajo social... trabajo social está abajo de la rama de la rama, y trabajo social podría inferir mucho desde arriba, que tuvieran en el profesiograma subdirecciones, coordinaciones (E7-MB).

En ámbitos tradicionales de Trabajo Social como: salud, educación y sistemas de procuración e impartición de justicia, el perfil técnico ha sentado las bases más representativas en las percepciones que tienen diferentes actores sobre sus posibilidades de intervención. En el caso de médicos/as cuando se les preguntó "cuáles creían que eran los niveles educativos de estos profesionistas", el 58\% refirió que tienen licenciatura; el $22 \%$, técnico; el $15 \%$, no sabe, y el $7 \%$, posgrado, como se muestra en el Gráfico 3.

Gráfico 3. Nivel de estudios de las/los trabajadores sociales

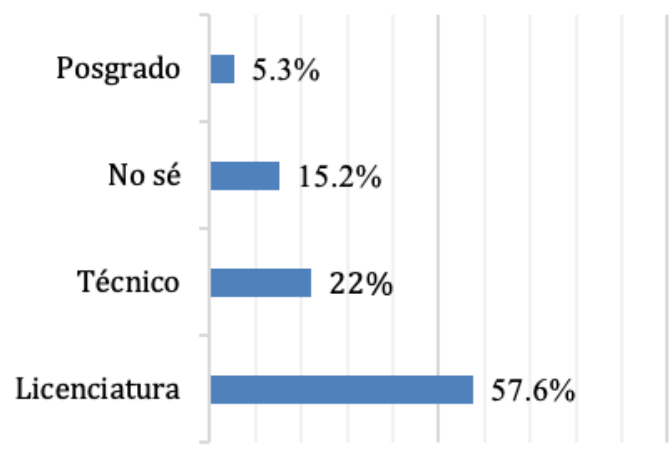

Fuente: Elaboración propia con base en los resultados del cuestionario.

Actualmente la plantilla cuenta con un importante número de personas con nivel técnico. Según el Manual de Organización del Departamento de Trabajo Social y Relaciones Públicas del Hospital General de México, 72 cuentan con licenciatura, y 39 son técnicos; mientras que en el caso de los estudios de posgrado hay una notable escasez, solo 5 tienen maestría, y no hay doctores titulados, solo estudiantes o pasantes que lo realizan en otro campo disciplinar. 
El nivel de estudios técnico es el de mayor tradición en la historia de esta carrera; de acuerdo con una médica de base con 21 años de experiencia en el Hospital General de México, la mayoría de trabajadores sociales eran profesionistas con estudios técnicos. En la actualidad este nivel de estudios ya no posee el mismo reconocimiento social al de grados superiores. De ahí que prevalezca una débil valoración y reconocimiento de su actividad por parte de médicos/as, al considerar que tienen bajos niveles educativos.

\section{Conclusiones}

Las/los médicos no tienen una actitud negativa o de rechazo hacia la práctica, los consideran un grupo importante en el movimiento hospitalario, con una alta carga de trabajo que resuelve diversos procesos administrativos y facilitan la estancia de los pacientes en el hospital. Sin embargo, no los reconocen como grupo de profesionales sanitarios que puedan aportar un conocimiento científico para mejorar la salud de los pacientes, sino como un personal administrativo.

Es claro que para las/los médicos, la práctica profesional de las/los trabajadores sociales no se destaca por saberes o conocimientos, sino por una actitud de servicio. Así, se identificaron las siguientes expresiones en torno a su comportamiento profesional: "mesurado, disponible, cooperador, resolutivo, generador de confianza, interesado en su trabajo, falta de exigencia, ausente y limitada demostración de su trabajo".

Durante las entrevistas con las/los médicos sobresalieron distintas expresiones relacionadas con: la importancia de la actividad de las/los trabajadores sociales; su invisibilidad $\mathrm{o}$ indiferencia hacia la función que realizan; además de juicios que califican este ejercicio desde una relación asimétrica o desigual, donde estos profesionistas ocupan una posición subalterna.

En ese sentido, Perales y Vizcaíno (2007) refieren que la estructuración de las representaciones sociales en torno a las actitudes no solo depende de las características del objeto, sino también del sistema social que instaura sus relaciones. Al respecto, la estructura del hospital está configurada en un orden jerárquico que expresa una clasificación de los grupos profesionales, según criterios de subordinación ba- sados en el capital social y las representaciones que los empleadores tengan de las disciplinas. Esta forma de organización de las profesiones sanitarias reconoce que existen disciplinas con saberes autónomos y subalternos.

Las/los trabajadores sociales ocupan una posición subordinada en la institución al desarrollar un ejercicio condicionado al diagnóstico médico, sin el cual no pueden actuar. En la estructura organizacional, solo tienen una jefatura, y no cuentan con su propia subdirección o área administrativa, y dependen de una subdirección médica en temas como: contratación de personal, horarios, actividades, recursos y otros. El escalafón que poseen no es un símbolo de autoridad para médicos/as, ya que ellos ocupan el primer rango en el organigrama. Esto se refleja en la forma como algunos médicos/as nombraron a las/los trabajadores sociales, "ayudantes o auxiliares", expresiones que refieren un servicio de asistencia y colaboración que les dan: "nos ayudan a conciliar con el mal carácter de los familiares...", "nos ayudan a agilizar los trámites administrativos o incluso a evaluar...", y "nos ayudan a ser resolutivos".

Los espacios de poder de las/los trabajadores sociales son pocos y se dan solo dentro de su departamento. Las autoridades del Hospital General de México les han encomendado principalmente la gestión administrativa de los servicios sociales, lo que ha contribuido en la protocolización de su función.

En consecuencia, la actitud de las/los médicos/as hacia el objeto no es unívoca, responde a las características del servicio, y sobre todo a sus experiencias con diferentes tipos de trabajadores sociales. Por un lado, presenta una orientación positiva al describirlos como: sensibles, empáticos y comprensivos con las necesidades de la población de bajo estrato socioeconómico, y se les otorga una carga afectiva-emotiva al identificarlos en la prestación de asistencia social. Pero por otro, una negativa debido a: un perfil bajo que poco evidencia sus habilidades; la carencia de rigor científico en sus interpretaciones; sus límites en su horario laboral (aunque la dinámica del hospital demanda mayor flexibilidad); o su ausencia en las discusiones con otros grupos profesionales.

Al respecto, las/los médicos describen la personalidad de las/los trabajadores sociales como "pasiva", por la timidez con la que actúan para demostrar la importancia de su actividad, rasgo que consideran como un factor influyente en el débil reconocimiento de su práctica. Aun- 
que las/los médicos destacan una buena actitud de servicioen las/los trabajadores sociales, difícilmente hacen otro tipo de distinción hacia su desempeño. Quienes les brindan un reconocimiento son los pacientes y sus familiares, gracias a los apoyos que les facilitan durante su estancia en la institución.

En definitiva, para que las/los trabajadores sociales tengan una participación distinta en ámbitos tradicionales como la salud, se sugiere reforzar la formación académica desde el nivel superior y generar especialidades en el campo sanitario, lo que probablemente impactará en la configuración de las representaciones sociales sobre su práctica profesional. La falta de especialización y defensa de su espacio profesional es quizás una consecuencia que ha provocado que terminen ciñéndose a modelos burocráticos $\mathrm{y}$, sean representados como parte de este aparato.

\section{Referencias bibliográficas}

Aylwin, N. (1999). Identidad e historia profesional. Revista Colombiana de Trabajo Social, 13, 7-23. Doi: https://doi.org/10.29344/07171714.8.1166

Banchs, M.A. (2000). Aproximaciones procesuales y estructurales al estudio de las Representaciones Sociales. Papers on Social Representations, 9. Recuperado de: http://www.psych.lse.ac.uk

Barrantes, C.A. (junio, 2003). Formación de Trabajadores Sociales en Venezuela. Hacia un estudio de representaciones sociales. Revista Servico Social, 5(2). Recuperado de: http://www.ssrevista.uel.br

Ducoing, P. (coord.) (2016). La investigación en educación: epistemologías y metodologías. D.F, México: AFIRSE- Plaza y Valdés.

Gómez, O., Sesma, S., Becerril, M., Knaul, M., Arreola, H. y Frenk, J. (julio 2011). Sistema de salud de México. Salud Pública de México, 2, 222-232. Recuperado de: http://www.scielo.org.mx.

Herzlich, C. (1975). La representación social: sentido del concepto. En: S. Moscovici, Introducción a la Psicología Social, (pp. 391-418). Barcelona, España: Plantea.

Hospital General de México. (18 de junio 2016). Manual de Organización del Departamento de Trabajo Social y Relaciones Públicas. Recuperado de: http://www.hgm.salud.gob.mx

Hospital General de México. (2016). Cuaderno estadístico enero-septiembre del HGM. Recuperado de http://www.hgm.salud.gob.mx

Jodelet, D. (1984). La representación social: fenómenos, concepto y teoría. En: S. Moscovici, Psicología Social II. Pensamiento y vida social Psicología social y problemas sociales (pp. 469-494). México, DF: Paidós.

Kvale, S. (2011). Las entrevistas en investigación cualitativa. Madrid: Morata.

López, R.E; Ribeiro, M; Cabello, M.L. (2007). “El Trabajo Social en México”. En Deslauriers, J.P. y Hurtubise, Y. (dirs.) El Trabajo Social Internacional. Elementos de comparación, (pp. 59-82). Buenos Aires, Argentina: Lumen-Humanitas.Lorente, B. y Luxardo, N. (marzo, 2018). Hacia una ciencia del trabajo social. Epistemologías, subalternidad y feminización. Cinta moebio: Revista de Epistemología de Ciencias Sociales, 61, 95-109. Doi: http://dx.doi.org/10.4067/S0717-554X2018000100095

Martínez, P. D. (julio-diciembre 2014). Aproximaciones hacia las representaciones sociales del trabajador social. Revista Perspectivas Sociales, 16(2), 63-79. Recuperado de: https://dialnet.unirioja.es/servlet/ articulo? codigo $=5072622$

Martínez, P. D (2019). Los médicos y sus representaciones sociales sobre las prácticas profesionales de los trabajadores sociales. México: Tesis de Doctorado. Facultad de Estudios Superiores Aragón, UNAM. Recuperado de: http://132.248.9.195/ptd2019/septiembre/0795489/Index.html

Minetto, V. y Voragini, N. (2017). Representaciones sociales sobre la intervención de los trabajadores sociales en educación, (Comunicación para el II Congreso de la Asociación Argentina de Sociología. I Jornadas de Sociología de la Universidad Nacional de Villa María. PRE ALAS, Las Ciencias Sociales en América Latina y el Caribe hoy. Perspectivas, debates y agendas de investigación, del 6 al 8 junio, Buenos Aires, Argentina).

Molineri, M. Esmoris, A.L., García, M., La Greca, N., Pérez, C., Brudnick, G., Rojas, M.S. y Etchandy, P. (diciembre, 2009). Algunas reflexiones sobre las representaciones sociales y prácticas de los profesionales de salud escolar. Facultad de Psicología-UBA/Secretaria de Investigaciones/Anuario de Investigaciones, 16, 231-237. Recuperado de: http://www.scielo.org.ar

Moscovici, S. (1979). El psicoanálisis, su imagen y su público. Buenos Aires: Huemul. 
Muriel, M., Martín, M. y García, T. (julio-diciembre, 2018). La imagen del trabajador social y los servicios sociales en la prensa escrita en España. Trabajo Social Global - Global Social Work, 8(15), 45-77. Doi: 10.30827/tsg-gsw.v8i15.7653

Organización Mundial de la Salud. (2017). ¿Cómo define la OMS la salud? Recuperado de: http://www. who.int

Perales, C.J. y Vizcaíno, M. (enero, 2007). Las relaciones entre actitudes y representaciones sociales: elementos para una integración conceptual. Revista Latinoamericana de Psicología, 2, 351-361. Recuperado de: http://www.scielo.org.co

Pérez, J.V y Bueno, J.R. (enero, 2005). Identidades y representaciones del Trabajo Social. Portularia revista de Trabajo Social, 5(2), 165-173. Recuperado de: http://dialnet.unirioja.es

Pérez, L; Díaz, Y.C; y Páez, G.I. (2013). Intervención social: representaciones sociales y prácticas de estudiantes de trabajo social. Análisis, 1(14), 20-40. Recuperado de: https://dialnet.unirioja.es

Rosacher, M.C (septiembre, 2015). Acerca de las representaciones sociales de la intervención del trabajador social. Así como nos miran, ¿nosotros miramos? Margen,78, 1-15. Recuperado de: https://dialnet. unirioja.es

Secretaría de Salud. (1 de junio 2017). Datos abiertos de México. Recuperado de: https://www.gob.mx

Torres, T.M., Maheda, M.E. y Aranda, C. (junio, 2004). Representaciones sociales sobre el psicólogo: investigación cualitativa en el ámbito de la formación de profesionales de la salud. Revista de Educación y Desarrollo. 2, 29-42. Recuperado de:http://www.cucs.udg.mx

Vázquez, S. y Cid de León, B.G. (junio, 2015). Sistematizando la acción del trabajo social en salud. Interacción y Perspectiva Revista de Trabajo Social, 1, 85-102. Recuperado de: http://www.produccioncientifica.luz.edu.ve 